\title{
SUCCESSIVE DIFFERENCES OF BOUNDED SEQUENCES
}

D. J. NEWMAN

Let $x_{0}, x_{1}, x_{2}, \cdots$ be a bounded sequence and write $\Delta x_{0}=x_{1}-x_{0}$, $\Delta^{2} x_{0}=x_{2}-2 x_{1}+x_{0}, \cdots$ and in general

$$
\Delta^{n} x_{0}=\sum_{k=0}^{n}(-1)^{k}\left(\begin{array}{l}
n \\
k
\end{array}\right) x_{n-k} .
$$

Of course if $\Delta^{n} x_{0} \equiv 0$ from some point on then $\left\{x_{n}\right\}$ is a constant sequence. On the other hand $\Delta^{n} x_{0}$ can approach 0 very rapidly without the sequence being a constant, e.g. if $x_{n}=1 / 2^{n}$ then $\Delta^{n} x_{0}=\left(-\frac{1}{2}\right)^{n}$. We will determine the exact rapidity with which $\Delta^{n} x_{0}$ can go to 0 for a nonconstant sequence.

THEOREM. A. Let $c>0$. There exists a nonconstant bounded sequence $\left\{x_{n}\right\}$ with $\left|\Delta^{n} x_{0}\right| \leqq(c / n)^{n}$.

B. Let $\left\{x_{n}\right\}$ be a bounded sequence for which $n\left|\Delta^{n} x_{0}\right|^{1 / n} \rightarrow 0$. Then $\left\{x_{n}\right\}$ is a constant.

Proof. A. Choose

$$
x_{n}=\sum_{k=0}^{n} \frac{(-\delta)^{k}}{k !}\left(\begin{array}{l}
n \\
k
\end{array}\right)
$$

so that $\Delta^{n} x_{0}=(-\delta)^{n} / n !$ and $\left|\Delta^{n} x_{0}\right| \leqq(c / n)^{n}$ when $\delta=c / e$. This sequence is certainly nonconstant and we need only show that it is bounded. We have

$$
\sum_{k=0}^{n} \frac{(-\delta)^{k}}{k !}\left(\begin{array}{l}
n \\
k
\end{array}\right)=1 / 2 \pi i \int_{C} e^{-\delta z}(1+1 / z)^{n} d z / z
$$

where $C$ is the circle $|z|=(n / \delta)^{1 / 2}$.

Write $z=(n / \delta)^{1 / 2} e^{i \theta}$ and observe that

$$
\begin{aligned}
e^{-\delta / n} \mid e^{-\delta z / n}(1+ & 1 / z)\left.\right|^{2} \\
& =e^{-\delta / n} \exp \left[-2(\delta / n)^{1 / 2} \cos \theta\right]\left(1+2(\delta / n)^{1 / 2} \cos \theta+\delta / n\right) \\
& \leqq 1,
\end{aligned}
$$

since $e^{-t}(1+t) \leqq 1$ for all real $t$. It follows immediately that $\left|e^{-\delta z}(1+1 / z)^{n}\right| \leqq \exp [\delta / n \cdot n / 2]=e^{\delta / 2}$ and so by (1) we obtain the bound $\left|x_{n}\right| \leqq e^{\delta / 2}$.

Received by the editors October 18, 1965. 
B. Write $F(z)=e^{-z} \sum x_{n}\left(z^{n} / n !\right)$ and note that along the positive axis

$$
|F(z)| \leqq e^{-z} \sum\left|x_{n}\right| \frac{z^{n}}{n !} \leqq e^{-z} C e^{z}=C
$$

Also

$$
F(z)=\sum \Delta^{n} x_{0} \frac{z^{n}}{n !}
$$

and by hypothesis for every $\epsilon>0$ a $C_{\epsilon}$ can be found so that $\left|\Delta^{n} x_{0}\right|$ $\leqq C_{\epsilon}\left(\epsilon^{2} / 2 n\right)^{n}$. Hence, for all $z$,

$$
\begin{aligned}
|F(z)| & \leqq C_{\epsilon} \sum\left(\frac{\epsilon^{2}}{2 n}\right)^{n} \frac{|z|^{n}}{n !} \leqq C_{\epsilon} \sum \frac{\left(\epsilon|z|^{1 / 2}\right)^{2 n}}{(2 n)^{n} n !} \\
& \leqq C_{\epsilon} \sum \frac{\left(\epsilon|z|^{1 / 2}\right)^{2 n}}{2 n !}
\end{aligned}
$$

so that

$$
|F(z)| \leqq C_{\epsilon} \exp \left[\epsilon|z|^{1 / 2}\right]
$$

The proof is now completed by an application of the PhrágmenLindelöff theorems.

We quote Titchmarch [1]:

Let $f(z)$ be analytic in $|\arg z|<\pi / 2 \alpha$, continuous in $|\arg z| \leqq \pi / 2 \alpha$. Suppose that $|f(z)| \leqq C$ on $|\arg z|=\pi / 2 \alpha$ and that $|f(z)|$ $\leqq C_{\epsilon} \exp \left[\epsilon|z|^{\alpha}\right]$ for every $\epsilon>0$ in $|\arg z|<\pi / 2 \alpha$. Then $|f(z)| \leqq C$ throughout $|\arg z| \leqq \pi / 2 \alpha$.

Applying this to $f(z)=F(-z), \alpha=\frac{1}{2}$ we conclude by (2) and (3) that $|F(-z)| \leqq C$ throughout the plane so that, since $F$ is entire, it is a constant. Thus $e^{-z} \sum x_{n}\left(z^{n} / n !\right)=C$ and so $x_{n}=C$.

\section{REFERENCE}

1. E. C. Titchmarch, The theory of functions, 2nd ed., Oxford Univ. Press, New York, 1961 ; p. 178.

Yeshiva UnIVERSITy 